\title{
Risk Factors Associated With Contrast-Induced Nephropathy after Primary Percutaneous Coronary Intervention
}

Dileep Kumar ${ }^{1}$, Hussain Liaquat ${ }^{1}$, Jawaid A. Sial ${ }^{1}$, Tahir Saghir ${ }^{1}$, Rekha Kumari ${ }^{2}$, Hitesh Kumar ${ }^{2}$, Musa Karim $^{3}$, Kelash Rai ${ }^{4}$, Reeta Bai ${ }^{5}$

1. Cardiology, National Institute of Cardiovascular Diseases, Karachi, PAK 2. Medical Officer, Government of Sindh, Mithi, PAK 3. Research, National Institute of Cardiovascular Diseases, Karachi, PAK 4. Internal Medicine, Wayne State University, Detroit, USA 5. Radiology, Dow University of Health Sciences, Karachi, PAK

Corresponding author: Dileep Kumar, dileep_dewani2011@yahoo.com

\section{Abstract \\ Background}

Contrast-induced nephropathy (CIN) after primary percutaneous coronary intervention (PCI) is associated with increased mortality and morbidity. The aim of this study is to determine the frequency of CIN after primary PCI and its association with risk factors in patients with ST-segment elevation myocardial infarction (STEMI) at a tertiary care cardiac center in Pakistan.

\section{Methodology}

In this observational study, we included 282 patients who presented with STEMI and underwent primary PCI at the National Institute of Cardiovascular Disease, Karachi, Pakistan, from October 2017 to April 2018. The serum creatinine $(\mathrm{mg} / \mathrm{dL})$ levels were obtained at baseline and 48 to 72 hours after the primary PCI procedure, and patients with a $25 \%$ increase or $\geqslant 0.5 \mathrm{mg} / \mathrm{dL}$ rise in post-procedure creatinine level (after 48 to 72 hour) were categorized for CIN.

\section{Results}

Out of a total sample of 282 patients, $68.4 \%$ (193) were males, and the mean age was $56.4 \pm 9.1$ years. A majority of the patients, $78.7 \%$ (222), were hypertensive and $34 \%$ (96) were diabetic. The CIN was observed in $13.1 \%$ (37) of the patients, and increased risk of CIN was found to be associated with the presence of diabetes mellitus and increased $(>200 \mathrm{~mL}$ ) use of contrast during the procedure, with odds ratios of 2.3 (1.14-4.63) and 3.12 (1.36-7.17), respectively.

\section{Conclusions}

Received 07/23/2020 Review began $07 / 25 / 2020$ Review ended 08/03/2020 Published 08/13/2020

\section{(c) Copyright 2020}

Kumar et al. This is an open access article distributed under the terms of the Creative Commons Attribution License CC-BY 4.0., which permits unrestricted use, distribution, and reproduction in any medium, provided the original author and source are credited.

The CIN after PCI is a common complication associated with the presence of diabetes mellitus and the use of an increased amount of contrast during the procedure.

Categories: Cardiology, Nephrology

Keywords: contrast-induced nephropathy, st-segment elevation myocardial infarction, primary percutaneous coronary intervention, acute kidney injury

\section{Introduction}

Primary percutaneous coronary intervention (PCI) is the recommended and preferred reperfusion strategy for the patients with ST-segment elevation myocardial infarction (STEMI). When performed within the 12hour window of symptom onset, it is an effective treatment strategy associated with a significant reduction in mortality and morbidity as compared to thrombolysis [1]. A decrease in glomerular filtration rate after procedure due to injection of contrast media, known as contrast-induced nephropathy (CIN), remains a real risk and is associated with increased risk of mortality and morbidity, consequently leading to prolongation of hospital stay, increased utilization of resources, and increased healthcare cost [2,3]. In recent literature, the frequency of CIN after primary PCI is reported to range from $10.4 \%$ to $23.2 \%$ [2-8]. CIN is reported to be associated with increased in-hospital mortality rate after primary PCI, with around four to eightfold increased risk of in-hospital mortality $[2,9]$.

Despite widespread use of contrast agents in intervention and radiographic studies, the pathophysiology of CIN is complex and not fully understood and involves various mechanisms, such as oxidative stress, vasoconstriction, medullary ischemia, and allergic reactions to contrast media [3,10]. Various patient- and procedure-related factors have been observed to be reasons that can the exaggerate acute kidney injury (AKI), such as preexisting chronic kidney diseases (CKDs), diabetes, hemodynamic alterations, and volume depletion due to cardiogenic shock or heart failure, complex interventional procedures, and use of increased 
Potential preemptive strategies to minimize the CIN are risk stratification of high-risk individuals, optimization of volume status, monitoring of adequate hydrations and infusion of normal saline before and after the procedure, and use of minimum contrast amount during the procedure. Guidelines on various preventive nonpharmacological and pharmacologic agents are not clear, and there is poor concordance in the literature, and despite the varying degree of agreement, iso-osmolar contrast or nonionic low-osmolar contrast is the preferred media among the interventional cardiology $[13,14]$.

CIN after primary PCI and its associated risk factors widely vary from study to study, and very limited data on our population are available regarding this important clinical condition; therefore, in this study our aim is to determine the frequency of CIN after primary PCI and its association with risk factors in patients with STEMI at a tertiary care cardiac center in Pakistan.

\section{Materials And Methods}

In this observational study, we included patients who presented to the emergency department with STEMI within 12 hours of the onset of symptoms and received primary PCI for revascularization at the National Institute of Cardiovascular Disease, Karachi, Pakistan, during October 2017 to April 2018.

The consented patients who fulfilled the inclusion criteria of the study were included consecutively. The Inclusion criteria were either gender, between 30 and 70 years of age, and normal serum creatinine level $(<1.2 \mathrm{mg} / \mathrm{dL})$ at baseline. Hemodynamically unstable patients, such as patients in shock or Killip class IV or those with pre-existing CKD or end-stage renal disease were excluded from the study.

Baseline demographic characteristics, such as gender and age (years), and clinical characteristics, such as history of diabetes, hypertension, and smoking, were recorded for all the patients. Diagnosis of STEMI was made based on history and electrocardiographic (ECG) changes at presentation, such as history of typical chest pain lasting for more than half an hour and supported by the baseline ECG findings of ST-segment elevation. Patients with a history of taking anti-hypertensive and anti-diabetic medication for at least six months were categorized as hypertensive and diabetic, respectively. Patients currently smoking or with a history of smoking a pack per day for at least one year were categorized as smokers.

The serum creatinine $(\mathrm{mg} / \mathrm{dL})$ levels were obtained at baseline and 48 to 72 hours after the primary PCI procedure, and patients with a $25 \%$ increase or $\geqslant 0.5 \mathrm{mg} / \mathrm{dL}$ rise in post-procedure creatinine level (after 48 to 72 hours) were categorized for CIN. The primary PCI procedures were performed by the consultant cardiologists, and the amount of contrast $(\mathrm{mL})$ used during the procedure was also recorded.

Appropriate, frequency (percentages) or quantitative summary measures, such as mean \pm standard deviation and minimum and maximum, were reported for the qualitative and quantitative characteristics of the patients. Stratifications were made by demographic characteristics (such as gender and age), co-morbidities (such as hypertension, diabetes, and smoking), and procedural characteristics (such as among of contrast used during the procedure), and odds ratios (ORs) along with $95 \%$ confidence interval (CI) were computed and the chi-square test was applied. Data analysis was performed with the help of IBM SPSS Version 19 (IBM Corp., Armonk, USA), and the level of significance was set at 5\%.

\section{Results}

In the study sample of 282 patients, 68.4\% (193) were male patients, and the mean age of the study sample was $56.4 \pm 9.1$ years. Hypertension was the most common co-morbid condition, which was observed in $78.7 \%$ (222) patients. The mean serum creatinine level increased from $0.77 \pm 0.16 \mathrm{mg} / \mathrm{dL}$ at baseline to $1.06 \pm 0.19$ $\mathrm{mg} / \mathrm{dL}, \mathrm{p}<0.001$, after 48 to 72 hours of the procedure. The CIN was observed in $13.1 \%$ (37) patients. The demographic and clinical characteristics of the patients included in this study are reported in Table 1. 


\section{Cureus}

Characteristics

Total

Total (N)

282

Gender

Male

Female

Age (years)

30 to 55 years

56 to 70 years

Co-morbid conditions

Hypertension

Diabetes mellitus

Smoking

Baseline serum creatinine level (mg/dL)

Range (minimum to maximum)

Mean \pm SD

Serum creatinine level (mg/dL) after 48 to 72 hours

Range (minimum to maximum)

Mean $\pm S D$

$1.06 \pm 0.19$

Contrast amount used $(\mathrm{mL})$

Range (minimum to maximum)

Mean \pm SD

$\leq 200 \mathrm{~mL}$

$>200 \mathrm{~mL}$

CIN

Yes

No
0.5 to 1.1

$0.77 \pm 0.16$

0.8 to 1.8

$68.4 \%$ (193)

$31.6 \%$ (89)

$56.4 \pm 9.1$

$42.6 \%(120)$

$57.4 \%$ (162)

$78.7 \%$ (222)

$34 \%(96)$

$27.7 \%(78)$

50 to 250

$158.97 \pm 43.49$

$87.2 \%(246)$

$12.8 \%(36)$

$13.1 \%$ (37)

$86.9 \%(245)$

TABLE 1: Demographic and clinical characteristics of the patients included in this study

SD, standard deviation; CIN, contrast-induced nephropathy

In this study, we observed no statistically significant association between the CIN, and gender, age, presence of hypertension, and smoking status of the patients. However, increased risk of CIN was found to be associated with the presence of diabetes mellitus and increased $(>200 \mathrm{~mL}$ ) use of contrast during the procedure with ORs of 2.3 (1.14-4.63) and 3.12 (1.36-7.17), respectively. Rate of CIN by various patient demographic and clinical characteristics and associated ORs are presented in Table 2. 


\section{Cureus}

\begin{tabular}{|c|c|c|c|c|}
\hline Characteristics & Base (N) & CIN rate, \% (n) & OR (95\% Cl) & p-Value \\
\hline Total & 282 & $13.1 \%(37)$ & - & - \\
\hline \multicolumn{5}{|l|}{ Gender } \\
\hline Male & 193 & $15.5 \%(30)$ & \multirow{2}{*}{$2.16(0.91-5.12)$} & \multirow{2}{*}{0.076} \\
\hline Female ${ }^{\star \star}$ & 89 & $7.9 \%(7)$ & & \\
\hline \multicolumn{5}{|l|}{ Age (years) } \\
\hline 30 to 55 years & 120 & $12.5 \%(15)$ & \multirow{3}{*}{$0.91(0.45-1.84)$} & \multirow{2}{*}{0.791} \\
\hline 56 to 70 years ${ }^{\star \star}$ & 162 & $13.6 \%(22)$ & & \\
\hline \multicolumn{4}{|l|}{ Hypertension } & \\
\hline Hypertensive & 222 & $14.4 \%(32)$ & \multirow{2}{*}{$1.85(0.69-4.98)$} & \multirow{2}{*}{0.216} \\
\hline Non-hypertensive $^{\star \star}$ & 60 & $8.3 \%(5)$ & & \\
\hline \multicolumn{5}{|l|}{ Diabetes mellitus } \\
\hline Diabetic & 96 & $19.8 \%(19)$ & \multirow{3}{*}{$2.3(1.14-4.63)$} & \multirow{2}{*}{$0.017^{\star}$} \\
\hline Non-diabetic ${ }^{\star \star}$ & 186 & $9.7 \%(18)$ & & \\
\hline \multicolumn{4}{|l|}{ Smoking } & \\
\hline Smokers & 78 & $12.8 \%(10)$ & \multirow{2}{*}{$0.96(0.44-2.1)$} & \multirow{2}{*}{0.926} \\
\hline Non-smokers ${ }^{\star \star}$ & 204 & $13.2 \%(27)$ & & \\
\hline \multicolumn{5}{|c|}{ Contrast amount used (mL) } \\
\hline$>200 \mathrm{~mL}$ & 36 & $27.8 \%(10)$ & \multirow{2}{*}{$3.12(1.36-7.17)$} & \multirow{2}{*}{$0.005^{\star}$} \\
\hline$\leq 200 \mathrm{~mL}^{\star \star}$ & 246 & $11 \%(27)$ & & \\
\hline
\end{tabular}

\section{TABLE 2: Rate of contrast-induced nephropathy (CIN) by various patient demographic and}

clinical characteristics

$\mathrm{CIN}$, contrast-induced nephropathy; OR, odds ratio; $\mathrm{Cl}$, confidence interval

**Benchmark; *Significant at $5 \%$

\section{Discussion}

CIN after primary PCI is an important complication reported to be associated with increased risk of inhospital mortality, prolonged hospitalization, and post-procedure complications such as need of ventilator and major bleeding events [2]. In our study, $13.1 \%$ of the patients developed CIN after primary PCI, which was slightly higher than the reported incidence of $12.4 \%$ in the study by Batra et al. [2] and 10.2\% in the study by Ullah et al. [15] for our population. These findings are aligned with the recent international studies, which reported CIN after primary PCI ranging from $10.4 \%$ to $23.2 \%$ [2-8]. A study based on NCDR CathPCI Registry by Tsai et al. [16] reported acute kidney injury in $7.1 \%$ of the patients after PCIs.

In our study, we observed that increased risk of CIN is associated with patients and procedure-related characteristics such as diabetes and increased amount of contrast used during procedure. A positive relationship between increased incidence of CIN and increased amount of contrast agent during procedure has been documented by various past studies [17,18]. Batra et al. [2] and Tsai et al. [16] had same observations regarding the amount of contrast and diabetes mellitus. In their observations, other patientrelated characteristics such as female gender, CKD, hypertension, dyslipidemia, history of anemia, and congestive heart failure were significantly associated with the development of CIN after primary PCI. Also, patients who developed CIN had longer hospital stays, significantly higher in-hospital mortality rate, and increased post-procedure complications such as need of ventilator and bleeding $[2,6,16]$. It has been suggested that increased baseline serum creatinine can be taken as a surrogate marker of extensive and severe atherosclerosis, circulatory instability, and major adverse cardiovascular event [19]. 
preemptive preventive strategies, pharmacological or nonpharmacological, in these patients. In a multicenter clinical trial, Chong et al. [20] reported that 12-hour sustained sodium chloride pre-hydration regimen may prevent CIN. Jo et al. [21] in their study assessed high-dose atorvastatin for the prevention of CIN, but the evidence was not supportive of the role of high-dose atorvastatin in preventing CIN. Nawa et al. [22] reported that for patients with poor renal function, continuous intravenous infusion of nicorandil for 4 hours before and 24 hours after procedure strongly prevent CIN after elective PCI. Similarly, Firouzi et al. [23] argued prophylactic use of intravenous magnesium sulfate for the prevention of CIN.

In clinical setting, early recognition of patients at increased risk of development of CIN is crucial, and various risk stratification modalities have been proposed in various studies. Mehran et al. [24] developed a simple score for the stratification of patients at high risk of CIN that has been validated and used by various studies ever since [25]. Kurtul et al. [26] tested neutrophil-to-lymphocyte ratio (NLR) for the risk stratification and reported that increased NLR is an independent predictor of CIN among patients who underwent PCI for non-NSTEMI. Kurtul and Duran [27] reported that the fragmented QRS complex is an independent predictor of CIN after primary PCI in patients with STEMI. Similarly, Goussot et al. [5] reported potential use of $\mathrm{N}$-terminal fragment of pro B-type natriuretic peptide for the identification of at-risk patients, a good predictive value of CHA2DS2-VASC score for the identification of patients at higher risk of CIN after urgent PCI for acute coronary syndrome has been reported by Kurtul et al. [7], and Ozturk et al. [8] concluded that the logistic clinical syntax score can be used to improve the accuracy of risk stratification for the development of CIN.

\section{Conclusions}

In conclusion, CIN after primary PCI is a common complication, and patients with diabetes mellitus are at an increased risk of development of CIN. Use of an increased amount of contrast during primary PCI procedure significantly increases the risk of post-procedure CIN. Early risk stratification and preemptive preventive measures are needed in clinical practice to minimize the clinical burden of CIN after primacy PCI.

\section{Additional Information \\ Disclosures}

Human subjects: Consent was obtained by all participants in this study. Animal subjects: All authors have confirmed that this study did not involve animal subjects or tissue. Conflicts of interest: In compliance with the ICMJE uniform disclosure form, all authors declare the following: Payment/services info: All authors have declared that no financial support was received from any organization for the submitted work. Financial relationships: All authors have declared that they have no financial relationships at present or within the previous three years with any organizations that might have an interest in the submitted work. Other relationships: All authors have declared that there are no other relationships or activities that could appear to have influenced the submitted work.

\section{References}

1. Ibanez B, James S, Agewall S, et al.: 2017 ESC Guidelines for the management of acute myocardial infarction in patients presenting with ST-segment elevation: The Task Force for the management of acute myocardial infarction in patients presenting with ST-segment elevation of the European Society of Cardiology (ESC). Eur Heart J. 2018, 39:119-77. 10.1093/eurheartj/ehx393

2. Batra MK, Sial JA, Kumar R, Saghir T, Karim M, Rizvi NH, Qamar N: Contrast-induced acute kidney injury: the sin of primary percutaneous coronary intervention. Pak Heart J. 2018, 51:172-8.

3. Azzalini L, Spagnoli V, Ly HQ: Contrast-induced nephropathy: from pathophysiology to preventive strategies. Can J Cardiol. 2016, 32:247-55. 10.1016/j.cjca.2015.05.013

4. Jurado-Román A, Hernández-Hernández F, García-Tejada J, et al.: Role of hydration in contrast-induced nephropathy in patients who underwent primary percutaneous coronary intervention. Am J Cardiol. 2015, 115:1174-8. 10.1016/j.amjcard.2015.02.004

5. Goussot S, Mousson C, Guenancia C, et al.: N-terminal fragment of pro B-type natriuretic peptide as a marker of contrast-induced nephropathy after primary percutaneous coronary intervention for ST-segment elevation myocardial infarction. Am J Cardiol. 2015, 116:865-71. 10.1016/j.amjcard.2015.06.007

6. Liu YH, Liu Y, Zhou YL, et al.: Comparison of different risk scores for predicting contrast induced nephropathy and outcomes after primary percutaneous coronary intervention in patients with ST elevation myocardial infarction. Am J Cardiol. 2016, 117:1896-903. 10.1016/j.amjcard.2016.03.033

7. Kurtul A, Yarlioglues M, Duran M: Predictive value of CHA2DS2-VASC score for contrast-induced nephropathy after percutaneous coronary intervention for acute coronary syndrome. Am J Cardiol. 2017, 119:819-25. 10.1016/j.amjcard.2016.11.033

8. Ozturk D, Celik O, Erturk M, et al.: Utility of the logistic clinical syntax score in the prediction of contrastinduced nephropathy after primary percutaneous coronary intervention. Can J Cardiol. 2016, 32:240-6. 10.1016/j.cjca.2015.05.005

9. Lucreziotti S, Centola M, Salerno-Uriarte D, et al.: Female gender and contrast-induced nephropathy in primary percutaneous intervention for ST-segment elevation myocardial infarction. Int J Cardiol. 2014, 174:37-42. 10.1016/j.ijcard.2014.03.087

10. Persson P, Tepel M: Contrast medium-induced nephropathy: the pathophysiology. Kidney Int Suppl. 2006, 69:8-10. 10.1038/sj.ki.5000367

11. Silvain J, Collet JP, Montalescot G: Contrast-induced nephropathy: the sin of primary percutaneous 
coronary intervention?. Eur Heart J. 2014, 35:1504-6. 10.1093/eurheartj/ehu126

12. McCullough PA: Contrast-induced acute kidney injury. J Am Coll Cardiol. 2008, 51:1419-28.

10.1016/j.jacc.2007.12.035

13. Shoukat S, Gowani SA, Jafferani A, Dhakam SH: Contrast-induced nephropathy in patients undergoing percutaneous coronary intervention. Cardiol Res Pract. 2010, 2010:649164. 10.4061/2010/649164

14. Xu R, Tao A, Bai Y, Deng Y, Chen G: Effectiveness of N-acetylcysteine for the prevention of contrastinduced nephropathy: a systematic review and meta-analysis of randomized controlled trials. J Am Heart Assoc. 2016, 5:003968. 10.1161/JAHA.116.003968

15. Ullah I, Israr M, Ali U, Iqbal MA, Ahmad F, Awan ZA: Frequency of contrast induced nephropathy in patients undergoing percutaneous coronary intervention. Pak Heart J. 2016, 48:130-3.

16. Tsai TT, Patel UD, Chang TI, et al.: Contemporary incidence, predictors, and outcomes of acute kidney injury in patients undergoing percutaneous coronary interventions: insights from the NCDR Cath-PCI registry. JACC Cardiovasc Interv. 2014, 7:1-9. 10.1016/j.jcin.2013.06.016

17. Chen SL, Zhang J, Yei F, et al.: Clinical outcomes of contrast-induced nephropathy in patients undergoing percutaneous coronary intervention: a prospective, multicenter, randomized study to analyze the effect of hydration and acetylcysteine. Int J Cardio. 2008, 126:407-13. 10.1016/j.ijcard.2007.05.004

18. Kane GC, Doyle BJ, Lerman A, Barsness GW, Best PJ, Rihal CS: Ultra-low contrast volumes reduce rates of contrast-induced nephropathy in patients with chronic kidney disease undergoing coronary angiography. J Am Coll Cardiol. 2008, 51:89-90. 10.1016/j.jacc.2007.09.019

19. Dangas G, Iakovou I, Nikolsky E, et al.: Contrast-induced nephropathy after percutaneous coronary interventions in relation to chronic kidney disease and hemodynamic variables. Am J Cardiol. 2005, 95:13-9. 10.1016/j.amjcard.2004.08.056

20. Chong E, Poh KK, Lu Q, et al.: Comparison of combination therapy of high-dose oral N-acetylcysteine and intravenous sodium bicarbonate hydration with individual therapies in the reduction of Contrast-induced Nephropathy during Cardiac Catheterisation and Percutaneous Coronary Intervention (CONTRAST): a multi-centre, randomised, controlled trial. Int J Cardiol. 2015, 201:237-42. 10.1016/j.ijcard.2015.07.108

21. Jo SH, Hahn JY, Lee SY, et al.: High-dose atorvastatin for preventing contrast-induced nephropathy in primary percutaneous coronary intervention. J Cardiovasc Med. 2015, 16:213-9. 10.2459/JCM.0000000000000157

22. Nawa T, Nishigaki K, Kinomura Y, Tanaka T, Yamada Y, Kawasaki M, Minatoguchi S: Continuous intravenous infusion of nicorandil for 4 hours before and 24 hours after percutaneous coronary intervention protects against contrast-induced nephropathy in patients with poor renal function. Int J Cardiol. 2015, 195:228-34. 10.1016/j.ijcard.2015.05.078

23. Firouzi A, Maadani M, Kiani R, et al.: Intravenous magnesium sulfate: new method in prevention of contrast-induced nephropathy in primary percutaneous coronary intervention. Int Urol Nephrol. 2015, 47:521-5. 10.1007/s11255-014-0890-z

24. Mehran R, Aymong ED, Nikolsky E, et al.: A simple risk score for prediction of contrast-induced nephropathy after percutaneous coronary intervention: development and initial validation. J Am Coll Cardiol. 2004, 44:1393-9. 10.1016/j.jacc.2004.06.068

25. Abellás-Sequeiros RA, Raposeiras-Roubin S, Abu-Assi E, et al.: Mehran contrast nephropathy risk score: is it still useful 10 years later?. J Cardiol. 2016, 67:262-7. 10.1016/j.jjcc.2015.05.007

26. Kurtul A, Yarlioglues M, Duran M, Murat SN: Association of neutrophil-to-lymphocyte ratio with contrastinduced nephropathy in patients with non-ST-elevation acute coronary syndrome treated with percutaneous coronary intervention. Heart Lung Circ. 2016, 25:683-90. 10.1016/j.hlc.2016.01.007

27. Kurtul A, Duran M: Fragmented QRS complex predicts contrast-induced nephropathy and in-hospital mortality after primary percutaneous coronary intervention in patients with ST-segment elevation myocardial infarction. Clin Cardiol. 2017, 40:235-42. 10.1002/clc.22651 